\title{
Endoscopic management of a sebaceous carcinoma arising in a mature cystic teratoma of the ovary
}

\author{
Daniele Bolla • Arthur von Hochstetter • René Hornung
}

Received: 19 September 2010 / Accepted: 28 October 2010/Published online: 11 November 2010

(C) Springer-Verlag 2010

\begin{abstract}
Malignant transformation of a mature cystic teratoma (MCT) of the ovary is extremely rare. Squamous cell carcinoma is the most common malignancy, which occurs in MCT and accounts for $\sim 80 \%$ of all cases. We describe the eighth undisputed case of a pure sebaceous carcinoma arising in a teratoma of the ovary. A 45-year-old woman underwent laparoscopic hysterectomy, bilateral salpingo-oophorectomy with pelvic and paraaortic lymphadenectomy. The final stage was pT1a, pN0 (0/30), M0, G2. The carcinoma was localized within a dermoid cyst not involving the capsule or adjacent abdominal structures. Complete tumor excision without iatrogenic tumor cell dissemination and proper staging are important for the prognosis and treatment and should be performed as soon as possible after diagnosis. Unfortunately, the very small number of cases published does not allow an evidencebased therapeutic management.
\end{abstract}

Keywords Mature cystic teratoma - Sebaceous carcinoma . Ovary $\cdot$ Endoscopic surgery

\section{Introduction}

Mature cystic teratomas (MCT) of the ovary, also known as dermoid cyst, represent $44 \%$ of ovarian neoplasms. They

\section{Bolla $(\bowtie) \cdot$ R. Hornung}

Department of Obstetrics and Gynecology,

Kantonsspital St. Gallen,

Rorschacher Strasse 95,

CH- 9007, St. Gallen, Switzerland

e-mail: daniele.bolla@kssg.ch

A. von Hochstetter

Pathology Institute Enge Zurich,

St. Gallen, Switzerland are benign tumors containing mature tissue of any of the three germ-cell layers. MCT represent up to $58 \%$ of benign ovarian tumors and typically occur in young women [4]. Malignant transformation is rare and occurs in $1-3 \%$ of cases [12]. It usually arises in postmenopausal women and can develop from any element of the dermoid cyst, but mainly from the ectodermal tissue. Squamous cell carcino$\mathrm{ma}$ is the most common malignancy that occurs in dermoid cysts and accounts for $75 \%$ to $88 \%$ of all cases $[6,9]$.

To the best of our knowledge, there are only seven reports of pure sebaceous carcinomas arising within a benign teratoma of the ovary $[1-3,6,7,11,12]$. We present the first case of this rare neoplasm managed by endoscopic surgery only.

\section{Case report}

A 45-year-old gravida 15 para 12 was referred to our hospital with a histological diagnosis of a sebaceous carcinoma within a mature cystic teratoma of the ovary and a TNM score of pT1a, pNX, M0, G2, and R0. Two months earlier, the patient had undergone a laparoscopic left-sided salpingo-oophorectomy in another clinic for an adnexal mass suspicious for a dermoid cyst. During this operation, the ovary was removed entirely using an endobag without rupturing the capsule. After the diagnosis of sebaceous carcinoma was rendered, an abdominal PET CT abdominal scan was performed. No metastatic lesions were found.

By admission in our hospital, physical examination revealed no peripheral lymphadenopathy or hepatosplenomegaly. The complete blood count was normal. The tumor marker CA125 was within a normal range. Past and family history was unremarkable. 


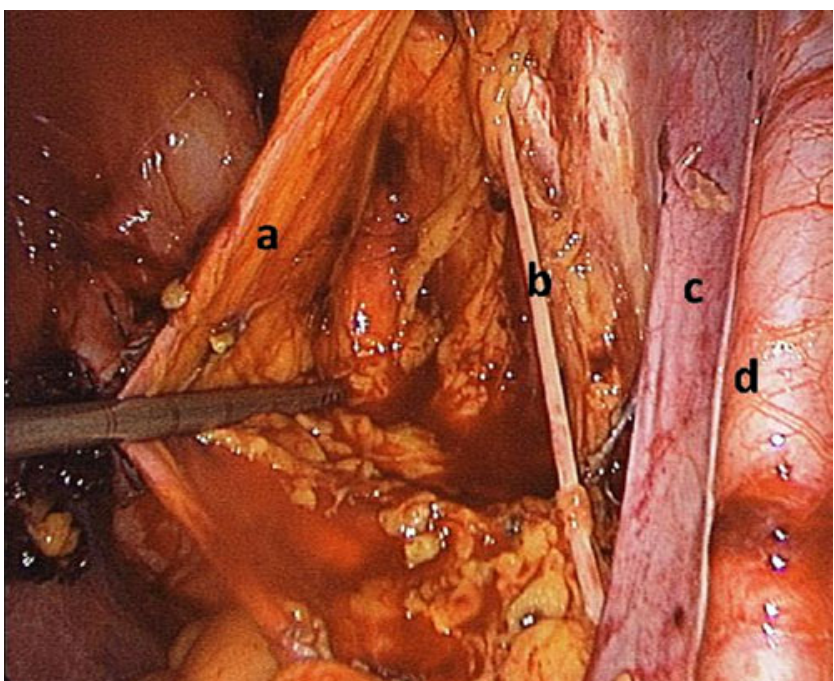

Fig. 1 Laparoscopic pelvic lymphadenectomy: a right lateral umbilical ligament, b right obturator nerve, $\mathbf{c}$ right external iliac vein, d right external iliac artery

An exploratory laparoscopic with surgical staging was undertaken. The uterus and the right adnexa were unremarkable. There was no ascites. No tumor implants were found in the peritoneal cavity. A total laparoscopic hysterectomy, right-sided salpingo-oophorectomy and laparoscopic pelvic and paraaortic lymphadenectomy were performed (Figs. 1, 2). The patient made an uncomplicated postoperative recovery.

At the tumor conference in our clinic we recommended no further treatment but only close follow-up gynecological examinations.

The patient is alive without evidence of recurrence 23 months after diagnosis.

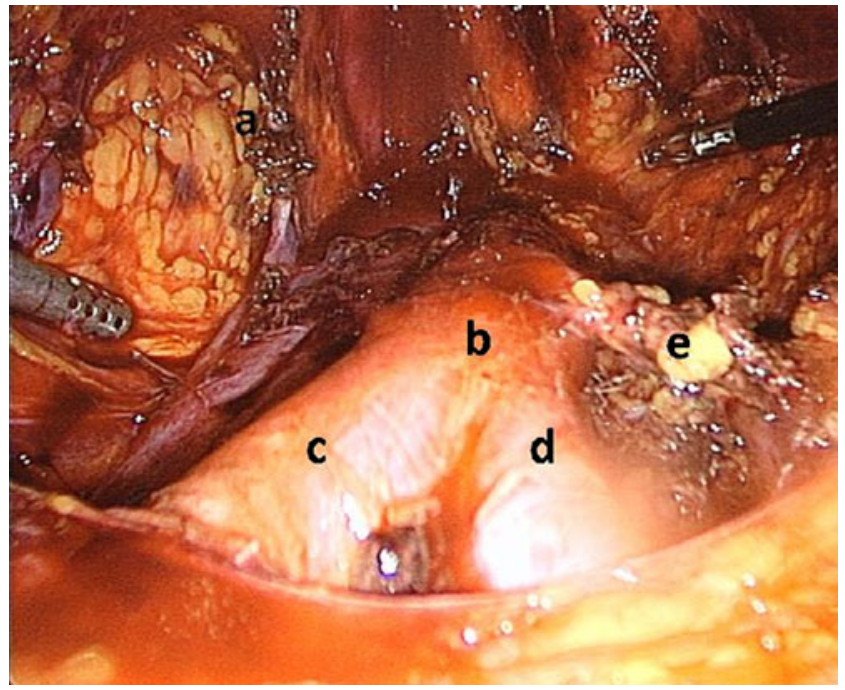

Fig. 2 Laparoscopic pelvic lymphadenectomy: a right ovarian vein, b aorta, $\mathbf{c}$ right common iliac artery, $\mathbf{d}$ left common iliac artery, e inferior mesenteric artery

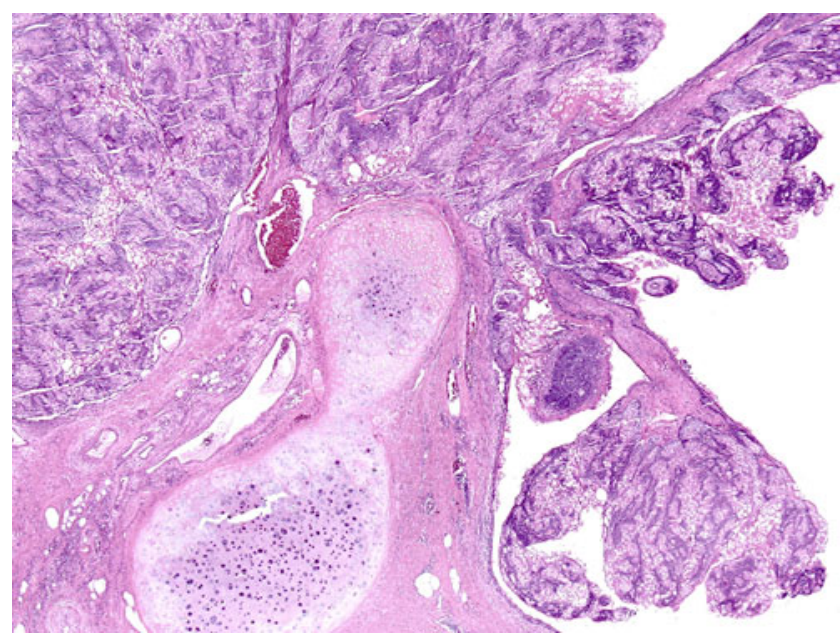

Fig. 3 Massive polypoid proliferation of basaloid epithelial cells with sebaceous differentiation within the lumen of a cystic teratoma. Nodules of mature cartilage tale up the base

\section{Pathological findings}

The cyst is lined by respiratory and squamous epithelium, the wall containing mature tissues including islands of cartilage, inflammatory infiltrates and foreign body giant cells.

In addition, the intraluminal tumor consists of polypoid confluent masses of dark basaloid cells showing varying degrees of sebaceous differentiation, the latter forming at times masses of parakeratotic debris (Fig. 3). The basaloid sheets contain prominent round to oval nuclei with finely granular chromatin and small- to medium-sized nucleoli. In some areas, nuclei and nucleoli are prominent and mitotic activity is brisk (Fig. 4). The pattern of growth is largely by pushing borders, but less circumscribed in areas, showing microscopic penetration into the ovarian stroma. Vascular invasion is not seen. The ovarian surface is not involved,

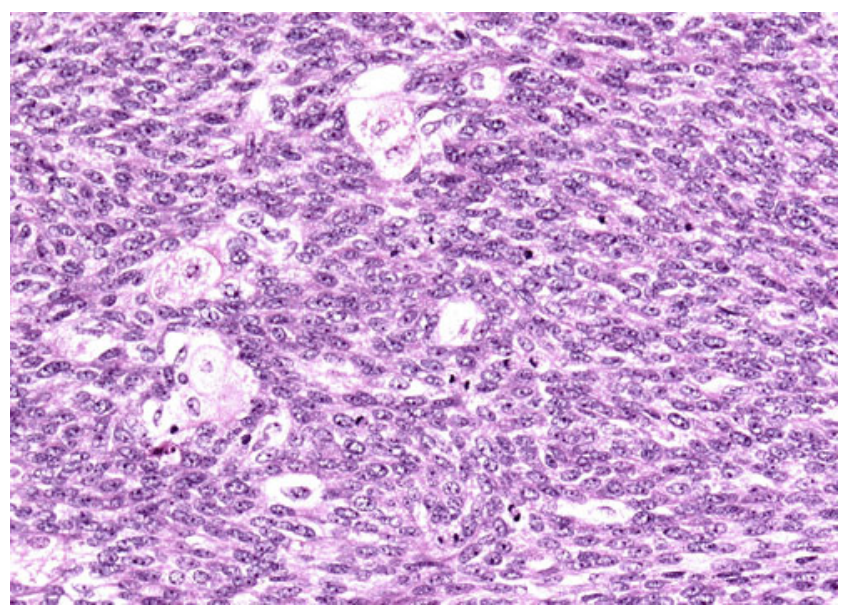

Fig. 4 In some areas, sebaceous differentiation was very focal, within sheets of poorly differentiated, highly proliferative basaloid cells 
intervening stroma remaining at least 2 to $3 \mathrm{~mm}$ wide. In analogy to sebaceous neoplasms of the skin, the features of hypercellularity, nuclear atypia, mitotic activity, and microinvasion were considered evidence of malignancy. Hence, the diagnosis of sebaceous carcinoma arising in a mature teratoma was rendered and subsequently confirmed in consultation by the AFIP (Washington, DC) and by Dr. A. Talerman (Thomas Jefferson University Hospital, Philadelphia). Tumor stage was determined as pT1a R0.

\section{Discussion}

Malignant transformation of a dermoid cyst of the ovary is rare and occurs in less than $3 \%$. It usually arises from the ectodermal tissues and occurs almost exclusively in postmenopausal women with a mean age of 50 years [11].

Squamous cell carcinoma is the most common component $(\sim 80 \%)$ in malignant transformation in teratomas. However, several other malignancies have also been reported.

We report the eighth undisputed case of a pure sebaceous carcinoma arising in a mature teratoma of the ovary. In our case, the carcinoma was localized in a dermoid cyst without involving the capsule or adjacent abdominal structures.

Teratomas with malignant transformation are usually larger than ordinary dermoid cysts $(10-20 \mathrm{~cm})$ [9]. Clinical findings are often unspecific like abdominal distension, lower abdominal pain associated with bowel or bladder symptoms. Notwithstanding this, a diagnosis of malignancy in teratoma can only rarely be made preoperatively [5].

The histogenesis of sebaceous carcinoma is still poorly understood. Some authors believe that they originate from pluripotent stem cells, while other suggested that they may originate from more differentiated sebaceous cells in mature teratomas, which undergo malignant transformaton rather than from stem cells [10].

In general, sebaceous carcinomas of the skin often cause regional metastasis, but seldom spread to viscera [10]. In fact, none of the seven sebaceous carcinomas reported in literature, in different stages at diagnosis, developed a recurrence or metastasis. This favorable outcome is supported by the findings by Ribeiro-Silva et al. of a low proliferation rate of $\mathrm{Ki} 67$ associated with an inactivation of p53 gene in the sebaceous carcinoma [9].

For this reason, the role of chemotherapy and radiotherapy in sebaceous carcinomas arising from teratomas is unclear. Neither postoperative radiation nor adjuvant chemotherapy in patients with extracapsular disease has shown to improve survival time.

Hence, debulking surgery remains crucial for patient survival. Petterson et al. described a 75\% 5-year survival rate for unruptured stage I tumors [8]. Kashimura et al. showed a $50 \% 5$-year survival rate in stage 1 versus $25 \%$ in stage 2 [5]. This referred to all types of teratomas with malignant transformation, showing that an improved surgical management of ovarian cancer is the single most important factor.

The utility of node dissection as well is controversial since the mode of spread is generally by direct extension or peritoneal seeding, but it may influence treatment planning, especially in early stage of disease.

In our case, the patient underwent laparoscopic hysterectomy, bilateral salpingo-oophorectomy with pelvic and paraaortic lymphadenectomy according the staging procedure for ovarian neoplasm (Figs. 1, 2 and 3). Omentectomie was omitted as peritoneal washing during the first surgery had been negative. The final stage was pT1a, pN0 (0/30), M0, G2. No further treatment was recommended. The patient is in excellent condition 23 month after diagnosis without evidence of local recurrence or metastasis.

Unfortunately, the very small number of cases published does not allow an evidence-based therapeutic management and prognosis. In our case, the therapeutic strategy proved effective. However, whether the patient was over-treated or not remains speculative.

In conclusion, a complete tumor excision and proper staging are important for prognosis and treatment planning and should be performed at the time of initial surgery or as soon as possible following pathologic diagnosis. In early tumor stage, a laparoscopic approach can be justified and midline laparotomy avoided.

Declaration of interest The authors report no conflicts of interest. The authors alone are responsible for the content and writing of the paper.

\section{References}

1. Betta PG, Cosimi MF (1984) Sebaceous carcinoma arising in benign cystic teratoma of the ovary. Case report. Eur J Gynaecol Oncol 5(2):146-149

2. Changchien CC, Chen L, Eng HL (1994) Sebaceous carcinoma arising in a benign dermoid cyst of the ovary. Acta Obstet Gynecol Scand 73(4):355-358

3. Chumas JC, Scully RE (1991) Sebaceous tumors arising in ovarian dermoid cysts. Int J Gynecol Pathol 10(4):356-363

4. Comerci JT Jr, Licciardi F, Bergh PA, Gregori C, Breen JL (1994) Mature cystic teratoma: a clinicopathologic evaluation of 517 cases and review of the literature. Obstet Gynecol 84(1):22-28

5. Dos Santos L, Mok E, Iasonos A, Park K, Soslow RA, Aghajanian C, Alektiar K, Barakat RR, Abu-Rustum NR (2007) Squamous cell carcinoma arising in mature cystic teratoma of the ovary: a case series and review of the literature. Gynecol Oncol 105(2):321-324, Epub 2007 Jan 22 
6. Kontogianni E, Koukoura E, Christopoulou E (2001) Squamous cell carcinoma arising in mature cystic teratoma of the ovary: a case report. Eur J Gynaecol Oncol 22(3):238-239

7. Papadopoulos AJ, Ahmed H, Pakarian FB, Caldwell CJ, McNicholas J, Raju KS (1995) Sebaceous carcinoma arising within an ovarian cystic mature teratoma. Int J Gynecol Cancer 5(1):76-79

8. Peterson WF (1957) Malignant degeneration of benign cystic teratomas of the overy; a collective review of the literature. Obstet Gynecol Surv 12(6):793-830

9. Ribeiro-Silva A, Chang D, Bisson FW, Ré LO (2003) Clinicopathological and immunohistochemical features of a sebaceous carcinoma arising within a benign dermoid cyst of the ovary. Virchows Arch 443(4):574-578, Epub 2003 Jun 27

10. Rim SY, Kim SM, Choi HS (2006) Malignant transformation of ovarian mature cystic teratoma. Int J Gynecol Cancer 16(1):140 144

11. Vartanian RK, McRae B, Hessler RB (2002) Sebaceous carcinoma arising in a mature cystic teratoma of the ovary. Int $\mathrm{J}$ Gynecol Pathol 21(4):418-421

12. Venizelos ID, Tatsiou ZA, Roussos D, Karagiannis V (2009) A case of sebaceous carcinoma arising within a benign ovarian cystic teratoma. Onkologie 32(6):353-355, Epub 2009 May 25 